\title{
Endoscopic snare polypectomy of a periampullary neoplasm
}

\section{G Kavinda Rajapakse ${ }^{1}$, ASD Liyanage ${ }^{2}$ and Mohan de Silva ${ }^{3}$}

A 65-year old man presented with a six-month history of loss of appetite. Clinical examination was unremarkable. Initial endoscopy was reported to be normal. Ultrasound scan of the abdomen showed a dilated common bile duct with no gall bladder calculi. Serum alkaline phosphatase was raised (630.2 U/L). Duodenoscopy with a side-viewing scope (TJF-160R Olympus) revealed

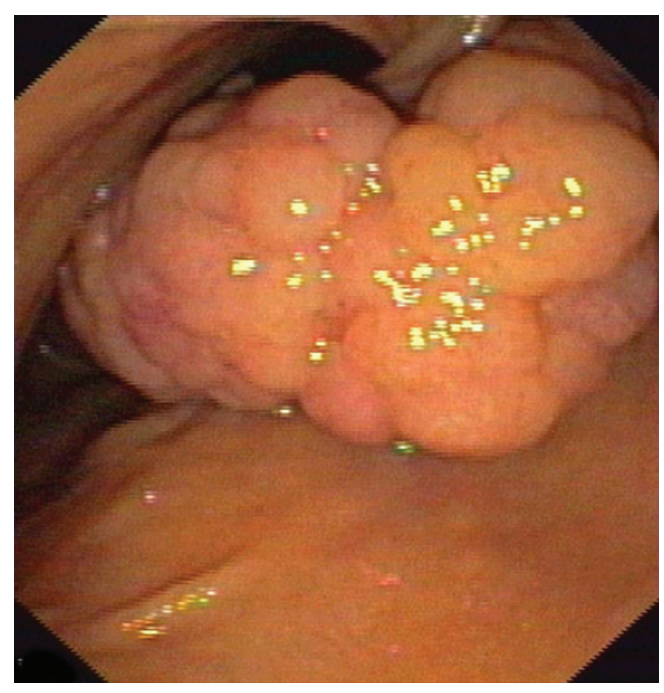

Figure 1

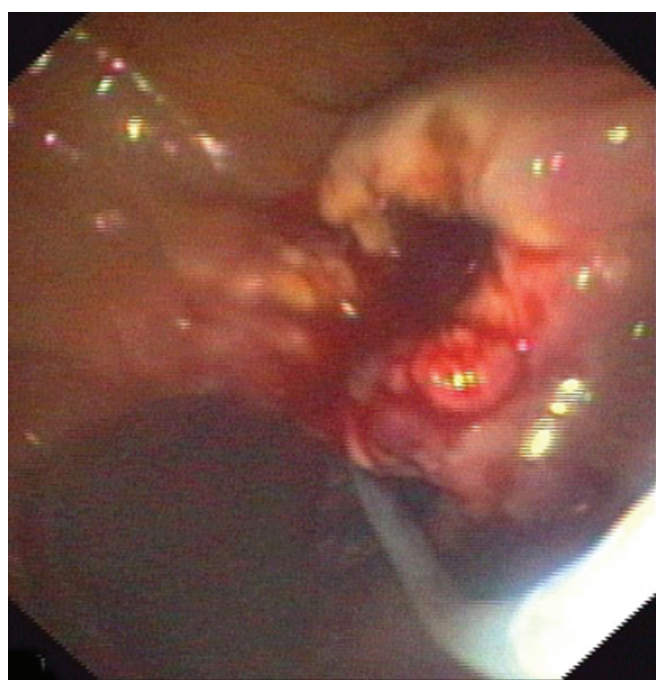

Figure 2 a periampullary growth hanging in to the duodenal lumen occluding the ampulla (Figure 1). Endoscopic snare polypectomy was performed. Free drainage of bile was observed after the polypectomy (Figure 2).

Histology showed a villous adenoma with moderate dysplasia. Excision margins were clear. Repeat endoscopic retrograde cholangio pancreaticogram after

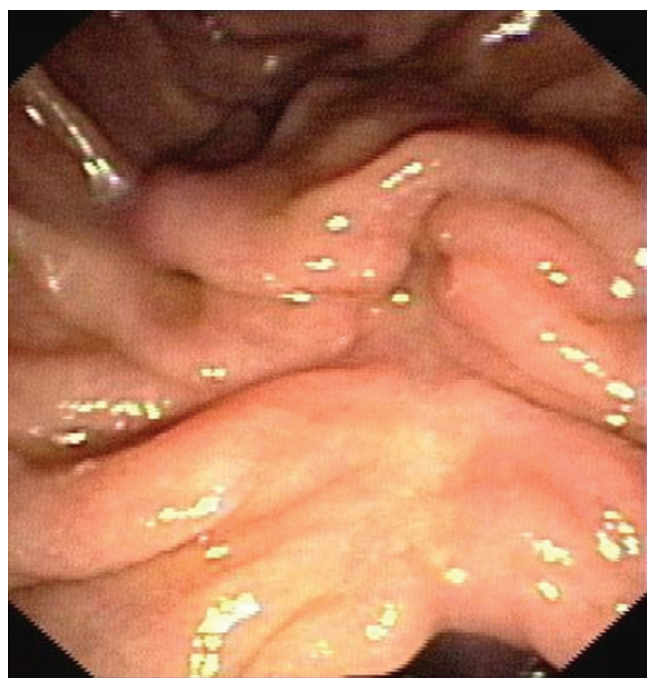

Figure 3

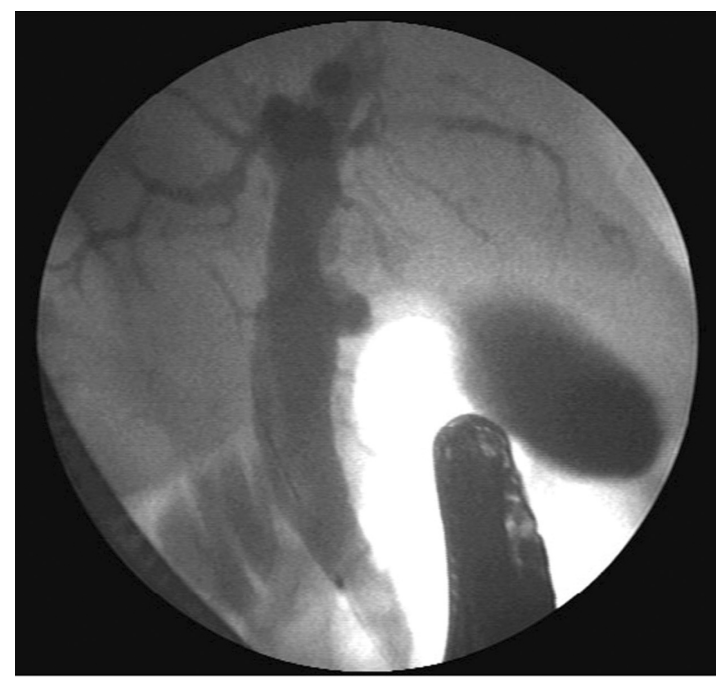

Figure 4

${ }_{1,2}$ Post-Intern House Officer, and ${ }^{3}$ Professor of Surgery, University Surgical Unit, Colombo South Teaching Hospital, Kalubowila, Sri Lanka.

Correspondence: MdeS, e-mail: <thathya@stmail.Ik> (Competing interests: none declared). Received 15 October 2005 and accepted 18 December 2005. 
one month showed a well healed ampulla (Figure 3). Bile duct was normal (Figure 4).

\section{Discussion}

Periampullary villous neoplasms may occur as isolated lesions or as part of a non-colonic spectrum of hereditary polyposis syndromes. As seen in this case, conventional endoscopy can miss prolapsing periampullary lesions. Duodenoscopy with a side-viewing duodenoscope is essential for diagnosis.

Tumours are amenable for endoscopic excision, endoscopic snare polypectomy is a simple minimal access technique for effective treatment. Surveillance endoscopy is needed for benign lesions with clear margins. 